\title{
Domain perturbation and invariant manifolds
}

\author{
NiCOLAS VARCHON
}

Abstract. We consider a reaction-diffusion equation defined on a sequence of bounded open sets $\left(\Omega_{n}\right)_{n \in \mathbb{N}}$, converging to $\Omega$ in the sense of Mosco, and we prove stability of invariant manifolds of the flux with respect to domain perturbation.

\section{Contents}

1. Introduction 547

2. Notations $\quad 549$

3. Local study of the flow 551

3.1. The Chow and Lu's theorem 551

3.2. Existence of local manifold for the perturbed flow 553

3.3. Extension of the manifolds 558

4. Stability of the manifolds $\quad 560$

4.1. Proof of Theorem 4.1: outline 560

4.2. Fundamental stability results $\quad 562$

4.3. Proof of Lemma 4.4 564

5. Some remarks 566

Open Access $\quad 568$

REFERENCES $\quad 568$

\section{Introduction}

Consider the following reaction-diffusion problem

$$
P_{\omega}\left(u_{0}\right):\left\{\begin{aligned}
u_{t}-\Delta u & =f(u) & & \text { in } \mathbb{R}^{+} \times \omega \\
u & =0 & & \text { on } \mathbb{R}^{+} \times \partial \omega \\
u(0) & =u_{0} & & \text { in } \omega,
\end{aligned}\right.
$$

Mathematics Subject Classification: 35B20, 35B35, 35B42, 35K20, 35 K57.

Keywords: Degenerate semigroups, Structural stability, Reaction-diffusion. 
where $\omega$ is a bounded open set in $\mathbb{R}^{N}$ without any smoothness conditions on the boundary, and $f$ is a function of class $C^{1}(\mathbb{R})$, globally Lipschitz continuous. The problem $P_{\omega}$ generates then a flux of solutions on $\mathbb{R}^{+}$. In this paper, we study the stability of this flux with respect to geometrical perturbations of the domain $\omega$. More precisely, we consider a sequence of open sets $\left(\Omega_{n}\right)_{n \in \mathbb{N}}$ uniformly included in $D$, where $D$ is a fixed ball. This sequence converges to an open set $\Omega \subset D$ in the sense of Mosco, defined by the following two conditions:

$$
\begin{aligned}
& \forall u \in H_{0}^{1}(\Omega), \quad \exists u_{n} \in H_{0}^{1}\left(\Omega_{n}\right) \text { such that } u_{n} \stackrel{H_{0}^{1}(D)}{\longrightarrow} u . \\
& \text { Let } u_{n_{k}} \in H_{0}^{1}\left(\Omega_{n_{k}}\right), \quad u_{n_{k}} \frac{H_{0}^{1}(D)}{k \rightarrow \infty} v \text {, then } v \in H_{0}^{1}(\Omega) .
\end{aligned}
$$

This convergence is exactly equivalent to the convergence of solutions of the elliptic problem [20]:

$$
\left\{\begin{aligned}
-\Delta u=f & \text { in } \omega, \\
u=0 & \text { on } \partial \omega .
\end{aligned}\right.
$$

This convergence is general enough, and in particular, it covers convergence used by Daners and Dancer in [12-14], which was defined in a geometrical way. For instance, in dimension two, if the number of connected components of $\Omega_{n}^{c}$ is uniformly bounded and if $\Omega_{n}^{c}$ converge to $\Omega^{c}$ for the Hausdorff metric, then $\Omega_{n}$ converge to $\Omega$ in the sense of Mosco [22]. In dimension $N>2$, this result has been generalized by a condition of flat cone on the boundary of $\Omega_{n}$ [7,8]. It is well known that if $\left(\Omega_{n}\right)_{n \in \mathbb{N}}$ converges to $\Omega$ in the sense of Mosco, the solutions $u_{n}$ of the system $P_{\Omega_{n}}\left(u_{0 n}\right)$ converge for all $T>0$ in $L^{2}\left((0, T), H_{0}^{1}(D)\right)$ to the solution $u$ of $P_{\Omega}\left(u_{0}\right)$ as soon as $\left(u_{0 n}\right)_{n \in \mathbb{N}}$ weakly converges in $L^{2}(D)$ to $u_{0}[13,14,21]$; here we agree to extend the function $u_{n}$ (respectively $u$ ) by zero outside $\Omega_{n}$ (resp. $\Omega$ ) with the same notation. Of course, this convergence is not uniform with respect to time. We are now interested in studying the stability of the structure of the flux when $t \rightarrow \infty$. It was noted by Dancer [13] that if the problem $P_{\Omega}\left(u_{0}\right)$ admits a hyperbolic point $\bar{u}$, then for $n$ large enough, $P_{\Omega_{n}}\left(u_{0 n}\right)$ admits a hyperbolic point $\bar{u}_{n}$, and the sequence of these stationary points converges to $\bar{u}$ with respect to $n$. Moreover, the stable and unstable manifolds associated to $\bar{u}_{n}$ converge for the Hausdorff metric to the manifolds - respectively stable and unstable - of $\bar{u}$. The works of Bates et al. [4-6] deal with existence and persistence of invariant manifolds for more general dynamical systems. Applied to our problem, this allows to prove persistence and stability of the local stable and unstable manifolds around one hyperbolic point $[4,5]$, and also persistence and stability of the local central unstable manifolds if the flux on the manifold is attractive or repulsive. The work of Kostin [19] on the invariant manifold for discrete semigroup implies in our situation persistence of local central unstable manifolds if we suppose existence of a stationary point for each problem $P_{\Omega_{n}}$, such that the sequence of those points converges to the stationary point of $P_{\Omega}$. 
In this paper, we suppose that $P_{\Omega}$ (.) has a stationary point $\bar{u}$, which is not necessarily hyperbolic, and we do not make any assumption on the problems $P_{\Omega_{n}}$. We know that there exists $\mathcal{M}$, a local central unstable manifold that contains $\bar{u}$ [18, Theorem 8.5.1], and we prove (Theorem 4.1) that for $n$ large enough, there exists a local central unstable manifold $\mathcal{M}_{n}$ for $P_{\Omega_{n}}$ such that the sequence $\mathcal{M}_{n}$ converges to $\mathcal{M}$ in the Hausdorff metric. Of course, there does not necessarily exist a sequence $\left(\bar{u}_{n}\right)_{n \in \mathbb{N}}$ of stationary points for $P_{\Omega_{n}}$, converging to $\bar{u}$. Using the argument of the proof of this result, we find again the result concerning the perturbed manifold for hyperbolic points, stated by Dancer.

The plan of this paper is as follows: in Sect. 2, we introduce the notation, in particular the extension operator and the degenerate semigroup. Section 3 is devoted to the application of the result of Chow and Lu [11] to the present situation. In Sect. 4, we present and prove the main result about stability of the manifold. Finally, Sect. 5 contains remarks and open problems.

\section{Notations}

Let $D$ be a ball in $\mathbb{R}^{N}$, which will contain all open sets considered here. Let $\omega$ be an open set. Since we consider perturbation of domains, it is necessary to extend the functions of $L^{2}(\omega)$ (respectively $\left.H_{0}^{1}(\omega)\right)$ to functions of $L^{2}(D)$ (resp. $H_{0}^{1}(D)$ ) so that all the solutions belong to the same space. Let $p$ be the canonic extension operator defined as

$$
p(v)(x)= \begin{cases}v(x) & \text { if } x \in \omega \\ 0 & \text { if } x \in D \backslash \omega .\end{cases}
$$

It is well-known that for all domains $\omega, p$ is an isometric continuous linear operator from $L^{2}(\omega)$ into $L^{2}(D)$ and from $H_{0}^{1}(\omega)$ into $H_{0}^{1}(D)$. Thus, it allows us to identify $L^{2}(\omega)$ (resp. $\left.H_{0}^{1}(\omega)\right)$ with a closed subspace of $L^{2}(D)$ (resp. $H_{0}^{1}(D)$ ) endowed with the induced topology. The convergence in the sense of Mosco (1) is then well defined. Let $B$ be a sectorial operator in $L^{2}(\omega)$ with its domain included in $H_{0}^{1}(\omega)$, see [18] for a definition. The operator $-B$ is then a generator of a semigroup $\mathrm{e}^{-B t}$. The resolvent operator of $B$ at $\lambda$, denoted by $R(\lambda, B)$, which is in $\mathcal{L}\left(L^{2}(\omega)\right)$, can be extended to an operator $R_{p}(\lambda, B)$ in $\mathcal{L}\left(L^{2}(D)\right)$ in the following way: let $f \in L^{2}(D)$ and $u=\left.R(\lambda, B) f\right|_{\omega}$ then $R_{p}(\lambda, B) f=p(u)$. Then we can define a new family of mappings $\{T(t)\}_{t \geq 0}$ in $\mathcal{L}\left(L^{2}(D)\right)$ by

$$
T(t)=\frac{1}{2 i \pi} \int_{\Gamma} \mathrm{e}^{\lambda t} R_{p}(\lambda, B) \mathrm{d} \lambda,
$$

where $\Gamma$ is a contour in $\rho(-B)$ with $\arg \lambda \rightarrow \pm \theta$ as $|\lambda| \rightarrow \infty$ for some $\theta \in\left(\frac{\pi}{2}, \pi\right)$. We call $\{T(t)\}_{t \geq 0}$ a degenerate semigroup [1]. Moreover, for all $u \in L^{2}(D)$, we have

$$
T(t) u=p\left(\left.\mathrm{e}^{-B t} u\right|_{\omega}\right) .
$$


In this paper, we will denote by $T(t)$ the degenerate semigroup of $B$. In the rest of this section and in Sect. 4, we will always identify $L^{2}(\omega)$ (resp. $H_{0}^{1}(\omega)$ ) with the closed subspace of $L^{2}(D)$ (resp. $H_{0}^{1}(D)$ ), and in order to simplify the notation, we will denote by $v$ the function $p(v)$, omitting the symbol $p$. On the contrary, in the Sect. 3, we will make the difference between the two notations in order to describe how we extend the manifolds into the space $H_{0}^{1}(D)$ (see 3.3).

We consider $-\Delta_{\omega}$ the Dirichlet Laplacian in its domain

$$
D\left(-\Delta_{\omega}\right)=\left\{u \in H_{0}^{1}(\omega) ; \quad \exists h \in L^{2}(\omega) \text { such that }-\Delta_{\omega} u=h \text { in } \omega\right\} .
$$

We denote by $R\left(\lambda,-\Delta_{\omega}\right)$ the corresponding resolvent operator at $\lambda$. We will call a solution to $P_{\omega}\left(u_{0}\right)$ a continuous function of $[0,+\infty)$ into $L^{2}(\omega)$, which satisfies in $(0,+\infty)$ the following integral equation:

$$
u(t)=\mathrm{e}^{\Delta_{\omega}(t)} u_{0}+\int_{0}^{t} \mathrm{e}^{\Delta_{\omega}(t-s)} f(u(s)) \mathrm{d} s .
$$

It is known that that for every $u_{0} \in L^{2}(\omega)$, there exists a unique solution $u\left(t, u_{0}\right)$ of $P_{\omega}\left(u_{0}\right)$ [18]. The flux of solutions is the application $S_{\omega}: \mathbb{R}^{+} \times L^{2}(\omega) \mapsto L^{2}(\omega)$ which to $\left(t, u_{0}\right)$ associates $S_{\omega}(t) x_{0}=u\left(t, x_{0}\right)$. Following the definition of Henry [18, Definition 6.1.1], $\mathcal{M} \subset L^{2}(\omega)$ will be called a local invariant manifold of the problem $P_{\omega}$ if for all $x_{0} \in \mathcal{M}$, there exists a solution $u($.) of (4) on an open interval $\left(t_{1}, t_{2}\right)$ containing 0 such that $u(0)=x_{0}$ and $u(t) \in \mathcal{M}$ for $t_{1}<t<t_{2}$. Sometimes $\mathcal{M}$ will be called invariant manifold of the flux of Eq. (4), or just of the flux (4). We will say that $u(t)$ is a solution of (4) on an interval $J$ of $\mathbb{R}$ if for all $t_{0}, t \in J, t_{0} \leq t$, $y(t)=u\left(t+t_{0}\right)$ is solution of (4) with $u_{0}=u\left(t_{0}\right)$.

The set of stationary solutions of the problem $P_{\omega}$, denoted by $S P(\omega)$, is the set of solutions in $H_{0}^{1}(\omega)$ of the elliptic equation

$$
-\Delta_{\omega} u=f(u)
$$

We will use in the sequel the notation

$$
c=\operatorname{Lip} f \text {. }
$$

If $\left(\Omega_{n}\right)_{n \in \mathbb{N}}$ is a sequence of open sets converging as $n \rightarrow \infty$ in the sense of Mosco to an open set $\Omega$ as we will consider in the sequel, then $R\left(\lambda,-\Delta_{\Omega_{n}}\right)$ converge to $R\left(\lambda,-\Delta_{\Omega}\right)$ in the uniform operator topology of $\mathcal{L}\left(L^{2}(D)\right)$ [10]. Also, for each $h \in L^{2}(D)$ we have:

$$
R\left(\lambda,-\Delta_{\Omega_{n}}\right) h \stackrel{H_{0}^{1}(D)}{\longrightarrow} R\left(\lambda,-\Delta_{\Omega}\right) h
$$

The compact injection of $H_{0}^{1}(\omega)$ into $H_{0}^{1}(D)$ guarantees that the spectral set of $\left(-\Delta, D_{\omega}(-\Delta)\right)$ is composed of eigenvalues, which can be arranged in a nondecreasing sequence

$$
\lambda_{1}(\omega) \leq \lambda_{2}(\omega) \leq \cdots \leq \lambda_{k}(\omega) \rightarrow+\infty
$$


where each eigenvalue is repeated according to its multiplicity. If we denote by $\mathcal{O}(D)$ the set of all open sets included in $D$, then for every $k \in \mathbb{N}$, the mappings $\lambda_{k}: \mathcal{O}(D) \mapsto \overline{\mathbb{R}}$ are continuous with respect to Mosco convergence $[9,10]$.

Let $J \subset \mathbb{R}$ be an interval. For any $\eta \in \mathbb{R}$ and any Banach space $E$, we denote by $C_{\eta}(J, E)$ the following Banach space

$$
C_{\eta}(J, E)=\left\{\varphi \mid \varphi: J \mapsto E \text { is continuous and } \sup _{t \in J} \mathrm{e}^{-\eta t}|\varphi(t)|_{E}<\infty\right\}
$$

with the norm

$$
|\varphi|_{C_{\eta}(J, E)}=\sup _{t \in J} \mathrm{e}^{-\eta t}|\varphi(t)|_{E}
$$

The distance between a point and a set $M$ of $E$ is given by

$$
d(x, M)=\inf _{y \in M}\|x-y\|_{E} .
$$

The ball in $E$ of center $x$ and radius $\rho$ will be denoted by $B_{E}(x, \rho)$. Let $\left(\varrho_{\rho}\right), 0<$ $\rho<\rho_{0}$ be the family of applications, which to a pair of sets $M_{1}, M_{2}$ in $E$ associates the real number

$$
\varrho_{\rho}\left(M_{1}, M_{2}\right)=\max \left\{\sup _{x \in M_{1} \cap \bar{B}_{E}(x, \rho)} d\left(x, M_{2}\right), \sup _{x \in M_{2} \cap \bar{B}_{E}(x, \rho)} d\left(x, M_{1}\right)\right\} .
$$

Let $\left(\rho_{i}\right)_{i \in \mathbb{N}}$ be a strictly increasing sequence that converges to $\rho_{0}$, the application $d_{B_{E}(x, \rho)}$ defined by

$$
d_{B_{E}(x, \rho)}\left(M_{1}, M_{2}\right)=\sum_{i=0}^{\infty} \frac{1}{2^{i}} \frac{\varrho_{\rho_{i}}\left(M_{1}, M_{2}\right)}{1+\varrho_{\rho_{i}}\left(M_{1}, M_{2}\right)} .
$$

is the distance between closed subsets of $B_{E}(x, \rho)$ for the topology induced from $E$.

The spaces $L^{2}(\omega)$ and $H_{0}^{1}(\omega)$ are endowed with their usual norms, and we denote by $(.,$.$) the scalar product in L^{2}(D)$.

\section{Local study of the flow}

\subsection{The Chow and Lu's theorem}

Given $\bar{u}$ a solution of the stationary problem $\bar{u} \in S P(\Omega)$, we shall prove now, using the result of Chow and $\mathrm{Lu}$ [11, Theorem 4.4], that there exists a local invariant manifold containing $\bar{u}$, and this manifold is the limit in the sense of Hausdorff of a sequence of local invariant manifolds for the dynamical system $P_{\Omega_{n}}$.

Let us recall the Chow and Lu result, restricted to our situation. Let $X, Y$ be Banach spaces such that $X$ is continuously embedded in $Y$ and let $S(t)$ be a strongly continuous semigroup of bounded operators on $Y$. Consider the following assumptions: 
$\left(H_{1}\right) \quad Y=Y_{1} \oplus Y_{2}$, where $Y_{1}$ and $Y_{2}$ are linear subspaces invariant under $S(t)$.

$\left(H_{2}\right) \quad P_{i} S(t) Y=S(t) Y_{i}$ where $P_{i}$ are the projections from $Y$ to $Y_{i}$.

$\left(H_{3}\right) \quad P_{i} Y$ and $P_{i} X$ are invariant under $S(t)$ and $S(t) Y \subset X$ for $t>0$.

$\left(H_{4}\right) S(t)$ can be extended to a group on $Y_{1}$.

$\left(H_{5}\right)$ There exist constants $\alpha, \beta, \eta, M$ and $M^{*}$ such that

$$
\begin{gathered}
\alpha>0, \quad \beta>0, \quad M \geq 1, \quad M^{*} \geq 0, \\
\left|\mathrm{e}^{-\eta t} S(t) P_{1} y\right|_{X} \leq M \mathrm{e}^{-\alpha t}|y|_{Y}, \quad \text { for } t \leq 0, \quad y \in Y, \\
\left|\mathrm{e}^{-\eta t} S(t) P_{2} x\right|_{X} \leq M \mathrm{e}^{-\beta t}|x|_{X}, \quad \text { for } t \geq 0, x \in X, \\
\left|\mathrm{e}^{-\eta t} S(t) P_{2} y\right|_{X} \leq\left(M t^{-\frac{1}{2}}+M^{*}\right) \mathrm{e}^{-\beta t}|y|_{Y}, \quad \text { for } t>0, y \in Y .
\end{gathered}
$$

Let $F \in C^{1}(X, Y)$ and consider the following integral equation

$$
x(t)=S(t) x_{0}+\int_{0}^{t} S(t-s) F(x(s)) \mathrm{d} s .
$$

Then $x\left(t, x_{0}\right)$ is the solution of (9), which is equal to $x_{0}$ at time $t=0$. We have the following result.

THEOREM 3.1 (Chow and Lu, see [11], Theorem 4.4). Let $\eta<0$. Assume that $\left(H_{1}\right)-\left(H_{5}\right)$ are satisfied, and let $K\left(\alpha, \beta, M, M^{*}\right)=M\left(\frac{1}{\alpha}+\frac{3}{\sqrt{\beta}}\right)+M^{*} \frac{1}{\beta}$. Suppose that

$$
\operatorname{Lip}(F) K\left(\alpha, \beta, M, M^{*}\right)<1 \text {. }
$$

Then there exists a $C^{1}$ invariant manifold $\mathcal{M}$ for the flow defined by (9) and $\mathcal{M}$ satisfies:

- $\mathcal{M}=\left\{x_{0}: x\left(t, x_{0}\right)\right.$ is defined for all $t \in \mathbb{R}^{-}$and $\left.P_{2} x\left(t, x_{0}\right) \in C^{0}\left(\mathbb{R}^{-}, X\right)\right\}$,

- $\mathcal{M}=\left\{\zeta+h(\zeta), \zeta \in P_{1} X\right\}$, where $h: P_{1} X \rightarrow P_{2} X$ is of class $C^{1}$.

REMARK 3.2. If $P_{1} X$ is an m-dimensional subspace of $X$, then the manifold $\mathcal{M}$ is of dimension equal to $m$.

REMARK 3.3. $K\left(\alpha, \beta, M, M^{*}\right)=M\left(\frac{1}{\alpha}+\frac{3}{\sqrt{\beta}}\right)+M^{*} \frac{1}{\beta}$ is such that the norm of the linear operator $\mathcal{F}: C_{\eta}\left(\mathbb{R}^{-}, Y\right) \rightarrow C_{\eta}\left(\mathbb{R}^{-}, X\right)$ defined by

$$
\mathcal{F} f=\int_{0}^{t} S(t-s) P_{1} f(s) \mathrm{d} s+\int_{-\infty}^{t} S(t-s) P_{2} f(s) \mathrm{d} s
$$

satisfies the estimate

$$
\|\mathcal{F}\| \leq K\left(\alpha, \beta, M, M^{*}\right) .
$$

Under the additional condition that

$$
\frac{M K\left(\alpha, \beta, M, M^{*}\right) \operatorname{Lip}(F)}{1-K\left(\alpha, \beta, M, M^{*}\right) \operatorname{Lip}(F)}<1,
$$

the manifold $\mathcal{M}$ is exponentially attractive [11, Theorem 5.1]. 


\subsection{Existence of local manifold for the perturbed flow}

We apply now the result of the previous section to our flow. Let $\bar{v}$ be any point in $D\left(-\Delta_{\omega}\right)$. For every $z_{0} \in L^{2}(\omega)$, the function $z\left(t, z_{0}\right)$ equal to $u\left(t, \bar{v}+z_{0}\right)-\bar{v}$ solves the integral equation

$$
z(t)=\mathrm{e}^{-L_{\omega}(t)} z_{0}+\int_{0}^{t} \mathrm{e}^{-L_{\omega}(t-s)}\left(g(z(s))+K_{\omega}\right) \mathrm{d} s .
$$

Here $L_{\omega}$ is the linearized operator at point $\bar{v}$ defined by

$$
L_{\omega}=-\triangle_{\omega}-f^{\prime}(\bar{v}), \quad D\left(L_{\omega}\right)=D\left(-\Delta_{\omega}\right),
$$

and $\mathrm{e}^{-L_{\omega} t}$ is the semigroup generated by $-L_{\omega}$. The mapping $g$ associates to $z \in H_{0}^{1}(\omega)$ the function $f(z+\bar{v})-f(\bar{v})-f^{\prime}(\bar{v}) z$, which belongs to $L^{2}(\omega) . K_{\omega}$ is equal to $\Delta_{\omega} \bar{v}+f(\bar{v})$. The operator $L_{\omega}$ is a sectorial operator in $L^{2}(\omega)$ [18] with compact resolvent $R\left(\lambda, L_{\omega}\right)$. The spectrum of $L_{\omega}$ again consists of eigenvalues, which can be arranged into a nondecreasing sequence $\left(\lambda_{k}\left(L_{\omega}\right)\right)_{k \in n \in \mathbb{N}}$ that are greater than $\lambda_{1}(\omega)-c$ (c is the Lipschitz constant of $f$ ). Recall that for all $\omega \subset D$, we have $\lambda_{1}(\omega) \geq \lambda_{1}(D)$, where $\lambda_{1}(D)$ is the first eigenvalue of the Dirichlet Laplacian on D. The semigroup $\mathrm{e}^{-L_{\omega} t}$ enjoys the following properties.

PROPOSITION 3.4. $\mathrm{e}^{-L_{\omega} t}$ is a strongly continuous semigroup of bounded operators which, for $x \in L^{2}(\omega), t>0$, and $\delta>0$, satisfies the following estimates

$$
\begin{aligned}
\left\|\left(\mathrm{e}^{-L_{\omega} t}-I\right) \mathrm{e}^{-L_{\omega} \delta} x\right\|_{L^{2}(\omega)} & \leq \frac{C_{1}}{\delta} t \mathrm{e}^{c(t+\delta)}\|x\|_{L^{2}(\omega)}, \\
\left\|\left(\mathrm{e}^{-L_{\omega} t}-I\right) \mathrm{e}^{-L_{\omega} \delta} x\right\|_{H_{0}^{1}(\omega)} & \leq \frac{C_{1}}{\delta} \sqrt{t+(1+c) t^{2}} \mathrm{e}^{c(t+\delta)}\|x\|_{L^{2}(\omega)},
\end{aligned}
$$

where $C_{1}=\max \left\{\frac{1}{\mathrm{e}}, \frac{\left|\lambda_{1}(D)-c\right|}{\lambda_{1}(D) \mathrm{e}}\right\}$.

Note that the constant $C_{1}$ is independent of the open set $\omega \in \mathcal{O}(D)$.

Proof. Since $L_{\omega}$ is a sectorial operator, the semigroup generated by $-L_{\omega}$ is strongly continuous and bounded for all $t \geq 0$ [18]. Let $\left(\mathrm{e}_{i}\right)_{i \in \mathbb{N}}$ be the sequence of eigenvectors of $L_{\omega}$ associated to the eigenvalue $\lambda_{i}=\lambda_{i}\left(L_{\omega}\right)$. In this basis, we write $x=\sum_{i \geq 1} x_{i} \mathrm{e}_{i}$. For all $\lambda>\lambda_{1}(D)-c$ and $t \geq 0$ we have

$$
\begin{aligned}
& \quad\left|\frac{\mathrm{e}^{-\lambda t}-1}{\lambda}\right| \leq t \mathrm{e}^{c t} \\
& \text { and }|\lambda| \mathrm{e}^{-\lambda t} \leq \frac{C_{1}}{t} \mathrm{e}^{c t} .
\end{aligned}
$$

We obtain the first inequality by writing that

$$
\left\|\left(\mathrm{e}^{-L t}-I\right) \mathrm{e}^{-L \delta} x\right\|_{L^{2}(\omega)}^{2} \leq \sup _{i \geq 1}\left|\frac{\mathrm{e}^{-\lambda_{i} t}-1}{\lambda_{i}}\right|^{2} \lambda_{i}^{2} \mathrm{e}^{-2 \lambda_{i} \delta}\|x\|_{L^{2}(\omega)}^{2} .
$$


To show the second one, recall that

$$
\left\|\nabla\left(\mathrm{e}^{-L t}-I\right) \mathrm{e}^{-L \delta} x\right\|_{L^{2}(\omega)}^{2}=\left(\left(L+f^{\prime}(\bar{v})\right)\left(\mathrm{e}^{-L t}-I\right) \mathrm{e}^{-L \delta} x,\left(\mathrm{e}^{-L t}-I\right) \mathrm{e}^{-L \delta} x\right)
$$

and that

$$
\left(L\left(\mathrm{e}^{-L t}-I\right) \mathrm{e}^{-L \delta} x,\left(\mathrm{e}^{-L t}-I\right) \mathrm{e}^{-L \delta} x\right)=\sum_{i \geq 1} \frac{\left(\mathrm{e}^{-\lambda_{i} t}-1\right)^{2}}{\lambda_{i}} \lambda_{i}^{2} \mathrm{e}^{-2 \lambda_{i} \delta} x_{i}^{2} .
$$

Then, we obtain the second inequality by combining the previous two equations and using the fact that $\left|\mathrm{e}^{-\lambda t}-1\right| \leq \mathrm{e}^{c t}$ for $t \geq 0$ and $\lambda>-c$.

Let $l$ be an integer such that $\lambda_{l-1}\left(L_{\omega}\right)<\lambda_{l}\left(L_{\omega}\right)$. The spectrum of $L_{\omega}$ can be written as a partition

$$
\sigma\left(L_{\omega}\right)=\sigma_{1} \cup \sigma_{2}, \quad \text { where } \sigma_{1}=\cup_{i=1}^{l-1} \lambda_{i}\left(L_{\omega}\right) .
$$

Let $P_{1}^{\omega}, P_{2}^{\omega}$ be the spectral projections associated to this decomposition [18]. The operator $P_{1}^{\omega}$ is nothing else than the orthogonal projection from $L^{2}(D)$ into its proper subspace generated by the $l-1$ first eigenvectors, and $P_{2}^{\omega}=I-P_{1}^{\omega}$. Let $Q_{1}^{\omega}, Q_{2}^{\omega}$ be the trivial extensions of those projections to $L^{2}(D)$, defined by

$$
\text { for } i=1,2, y \in L^{2}(D) \quad Q_{i}^{\omega}(y)=p\left(P_{i}^{\omega}\left(\left.y\right|_{\omega}\right)\right) \text {. }
$$

The following proposition states that the semigroup verifies the conditions required for the application of Chow and Lu's theorem.

PROPOSITION 3.5. Let $a, b \in \mathbb{R}$ be nonnegative such that $\lambda_{l-1}\left(L_{\omega}\right)<a<$ $b<\lambda_{l}\left(L_{\omega}\right)$. Then for all $\eta, \alpha, \beta$ satisfying $a<-\eta<b, 0<\alpha<-a-\eta$ and $0<\beta<b+\eta$, the semigroup $\mathrm{e}^{-L_{\omega} t}$ satisfies the conditions $\left(H_{1}\right)-\left(H_{5}\right)$ for $X=$ $H_{0}^{1}(\omega), Y=L^{2}(\omega)$ and $Y_{i}=P_{i}^{\omega} Y(i=1,2)$. Moreover, $M$ and $M^{*}$ depend only on the constants $a, b, c, \eta, \beta$ and $\lambda_{1}(D)$.

Proof. The first four assumptions come directly from the properties of the spectral projections [18]. We will show that the fifth one is also satisfied. In this proof, we omit the index $\omega$ to simplify the notations. We first note that for all $t \leq 0$

$$
\begin{aligned}
\left(L \mathrm{e}^{-L t} P_{1} y, \mathrm{e}^{-L t} P_{1} y\right) & \leq a \mathrm{e}^{-2 a t}\|y\|_{L^{2}(\omega)}^{2}, \quad \text { and } \\
\left(\mathrm{e}^{-L t} P_{1} y, \mathrm{e}^{-L t} P_{1} y\right) & \leq \mathrm{e}^{-2 a t}\|y\|_{L^{2}(\omega)}^{2} .
\end{aligned}
$$

We then obtain the first estimate of $\left(H_{5}\right)$ by using the fact that

$$
\left\|\mathrm{e}^{-L_{\omega} t} P_{1} y\right\|_{H_{0}^{1}(\omega)}^{2}=\left(\left(L+f^{\prime}(\bar{v})+1\right) \mathrm{e}^{-L t} P_{1} y, \mathrm{e}^{-L t} P_{1} y\right) .
$$

For the second one, we observe that for $t>0$

$$
\begin{aligned}
\left(L \mathrm{e}^{-L t} P_{2} y, \mathrm{e}^{-L t} P_{2} y\right) & \leq b \mathrm{e}^{-2 b t}\left(L_{\omega} P_{2} y, P_{2} y\right), \quad \text { and } \\
\left(\mathrm{e}^{-L t} P_{2} y, \mathrm{e}^{-L t} P_{2} y\right) & \leq \mathrm{e}^{-2 b t}\|y\|_{L^{2}(\omega)}^{2},
\end{aligned}
$$


and the result follows. For proving the last one, we recall the for all $\lambda>b$

$$
\lambda \mathrm{e}^{-(\eta+\lambda) t} \leq \frac{C(b, \eta, \beta)}{t} \mathrm{e}^{-\beta t}
$$

where $C(b, \eta, \beta)=\frac{b}{b+\eta-\beta}$. Hence

$$
\mathrm{e}^{-2 \eta t}\left(L \mathrm{e}^{-L t} P_{2} y, \mathrm{e}^{-L t} P_{2} y\right) \leq \frac{C(b, \eta, \beta)}{t} \mathrm{e}^{-2 \beta t}\|y\|_{L^{2}(\omega)}^{2}
$$

With the second inequality of (16), we obtain

$$
\mathrm{e}^{-\eta t}\left\|\mathrm{e}^{-L t} P_{2} y\right\|_{H_{0}^{1}(\omega)} \leq\left(\sqrt{\frac{C(b, \eta, \beta)}{t}}+\sqrt{1+c}\right) \mathrm{e}^{-\beta t}\|y\|_{L^{2}(\omega)}
$$

The estimates are then satisfied with

$$
M=\max \{\sqrt{1+c+a}, \sqrt{b+c+1}, \sqrt{C(b, \eta, \beta)}\}, \quad M^{*}=\sqrt{1+c} .
$$

REMARK 3.6. - Once the constants $a, b, c, \eta, \alpha, \beta$ are chosen, the terms $M, M^{*}$ are independent of the choice of $\omega$ and of $\bar{v}$ as soon as the following inequality is satisfied

$$
\lambda_{l-1}\left(L_{\omega}\right)<a<b<\lambda_{l}\left(L_{\omega}\right) .
$$

- If we substitute $T_{\omega}(t)$, the degenerate semigroup of $L_{\omega}$, to $\mathrm{e}^{-L_{\omega} t}$, replace the spaces $L^{2}(\omega), H_{0}^{1}(\omega)$ by $L^{2}(D), H_{0}^{1}(D)$, respectively, and the operators $P_{1}^{\omega}, P_{2}^{\omega}$ by $Q_{1}^{\omega}, Q_{2}^{\omega}$, respectively, then the conditions $\left(H_{2}\right),\left(H_{3}\right),\left(H_{5}\right)$ are still satisfied, with the same constants. The condition $\left(H_{1}\right)$ should then be written as: " $T_{\omega}(0) L^{2}(D)=Y_{1} \oplus Y_{2}$," and the condition $\left(H_{5}\right)$ : " $T_{\omega}(t)$ can be extended to a degenerate group" that is defined in the same way as the degenerate semigroup.

In order to prove existence of local invariant manifolds, we need to modify the Eq. (10) outside a neighborhood of $\bar{v}$. Let $\psi \in C_{c}^{\infty}(\mathbb{R})$ be a regularizing function such that:

$$
|\psi|_{L^{\infty}} \leq 1 \text { and } \psi(x)= \begin{cases}1 & \text { for }|x| \leq 1 \\ 0 & \text { for }|x| \geq 2\end{cases}
$$

For all $\rho>0$, we define the function $\psi_{\rho}$ by $\psi_{\rho}()=.\psi(\dot{\bar{\rho}})$ and the function $G_{\rho}$ by

$$
G_{\rho}(u)=\psi_{\rho}\left(\|u\|_{H_{0}^{1}(\omega)}\right) g(u) .
$$

As it will be proved in Lemma 3.11, $G_{\rho} \in C^{1}\left(H_{0}^{1}(\omega), L^{2}(\omega)\right)$ and $\operatorname{Lip}\left(G_{\rho}\right) \rightarrow 0$ as $\rho \rightarrow 0$. For all $\rho>0$, the integral equation

$$
w(t)=\mathrm{e}^{-L_{\omega}(t)} w_{0}+\int_{0}^{t} \mathrm{e}^{-L_{\omega}(t-s)}\left(G_{\rho}(w(s))+K_{\omega}\right) \mathrm{d} s
$$


defines a new flow that is equivalent to the flow defined by the Eq. (10) inside the ball $B_{H_{0}^{1}(D)}(0, \rho)$ in the sense that if $z_{0} \in B_{H_{0}^{1}(D)}(0, \rho)$, then for all $t>0$ such that $z\left(t, z_{0}\right) \in B_{H_{0}^{1}(D)}(0, \rho)$ where $w\left(t, z_{0}\right) \in B_{H_{0}^{1}(D)}(0, \rho)$, we have

$$
z\left(t, z_{0}\right)=w\left(t, z_{0}\right)
$$

where, as usually, $w\left(t, w_{0}\right)$ is the solution of (18) equal to $w_{0}$ at $t=0$. The next theorem states that the flux (18) has a $l$-1-dimensional global invariant manifold $\mathcal{M}_{\rho}$ for $l$, verifying the condition $\left(C_{1}\right)$ below and that the flow of $P_{\omega}$ has a $l-1$-dimensional local invariant manifold $\bar{v}+\mathcal{M}_{\rho}^{l o c}$.

THEOREM 3.7. Assume that $\omega$ and $\bar{v}$ are such that

$\left(C_{1}\right) \lambda_{l-1}\left(L_{\omega}\right)<a<b<\lambda_{l}\left(L_{\omega}\right)$

with $a, b$ nonnegative. If $\rho$ is small enough to have

$\left(C_{2}\right) \operatorname{Lip}\left(G_{\rho}\right) K\left(\alpha, \beta, M, M^{*}\right)<\frac{1}{2}$

then there exists $\mathcal{M}_{\rho}$, global $C^{1}$ invariant manifold for the flow (18), which satisfies:

$\left(G_{1}\right) \quad \mathcal{M}_{\rho}=\left\{w_{0}: w\left(t, w_{0}\right)\right.$ is defined for all $t \in \mathbb{R}^{-}$ and $\left.P_{2}^{\omega} w\left(t, w_{0}\right) \in C^{0}\left(\mathbb{R}^{-}, H_{0}^{1}(\omega)\right)\right\}$,

$\left(G_{2}\right) \quad \mathcal{M}_{\rho}=\left\{\zeta+h_{\omega}(\zeta), \zeta \in P_{1}^{\omega} L^{2}(\omega)\right.$,

$$
\text { where } \left.h_{\omega}: P_{1}^{\omega} H_{0}^{1}(\omega) \rightarrow P_{2}^{\omega} H_{0}^{1}(\omega) \text { is } C^{1}\right\} \text {, }
$$

Moreover, if $K_{\omega}$ is such that the following condition is satisfied

$\left(C_{3}\right)\left\|K_{\omega}\right\|_{L^{2}(\omega)}<\frac{\rho}{2 K\left(\alpha, \beta, M, M^{*}\right)}$,

Then the set $\mathcal{M}_{\rho}^{\text {loc }}$ defined by

$$
\mathcal{M}_{\rho}^{l o c}=\mathcal{M}_{\rho} \cap B_{H_{0}^{1}(\omega)}(0, \rho)
$$

is a nonempty local $C^{1}$ invariant manifold for the flow defined by the Eq. (10), and then $\bar{v}+\mathcal{M}_{\rho}^{\text {loc }}$ is a local invariant manifold of $P_{\omega}$.

REMARK 3.8. - We will show in Lemma 3.11 that the assumption $\left(C_{2}\right)$ is realized for $\rho$ small enough.

- We note that in the previous theorem, the manifolds $\mathcal{M}_{\rho}, \mathcal{M}_{\rho}^{\text {loc }}$ and $\bar{v}+\mathcal{M}_{\rho}^{\text {loc }}$ are $(l-1)$-dimensional.

REMARK 3.9. Existence of the manifold $\mathcal{M}_{\rho}$ is given by Theorem 3.1 above. In Chow and $\mathrm{Lu}$ [11], the manifold is constructed in the following way: for every $\zeta \in P_{1}^{\omega} L^{2}(\omega)$, we have $h_{\omega}(\zeta)=P_{2}^{\omega}(\varphi(\zeta)(0))$ where $\varphi(\zeta)$ is the unique fixed point belonging to $C_{\eta}\left(\mathbb{R}^{-}, H_{0}^{1}(\omega)\right)$ of the mapping $\mathcal{J}_{\omega}$ defined by

$$
\begin{aligned}
\mathcal{J}_{\omega}(\zeta, x)(t)= & \mathrm{e}^{-L_{\omega} t} \zeta+\int_{0}^{t} \mathrm{e}^{-L_{\omega}(t-s)} P_{1}^{\omega}\left(G_{\rho}(x(s))+K_{\omega}\right) \mathrm{d} s \\
& +\int_{-\infty}^{t} \mathrm{e}^{-L_{\omega}(t-s)} P_{2}^{\omega}\left(G_{\rho}(x(s))+K_{\omega}\right) \mathrm{d} s .
\end{aligned}
$$


The mapping $\mathcal{J}_{\omega}$ is a uniform contraction in $C_{\eta}\left(\mathbb{R}^{-}, H_{0}^{1}(\omega)\right)$ with respect to the variable $\zeta$ with a contraction coefficient smaller than $\operatorname{Lip}\left(G_{\rho}\right) K\left(\alpha, \beta, M, M^{*}\right)$. Hence for every $w_{0} \in \mathcal{M}_{\rho}$, there exists $w\left(t, w_{0}\right)$ solution of (18) in $\mathbb{R}^{-}$such that $w\left(t, w_{0}\right) \in$ $C_{\eta}\left(\mathbb{R}^{-}, H_{0}^{1}(D)\right)$ and $w\left(t, w_{0}\right)$ is a solution of the integral equation

$$
\begin{aligned}
w(t)= & \mathrm{e}^{-L_{\omega} t} P_{1}^{\omega} w_{0}+\int_{0}^{t} \mathrm{e}^{-L_{\omega}(t-s)} P_{1}^{\omega}\left(G_{\rho}(w(s))+K_{\omega}\right) \mathrm{d} s \\
& +\int_{-\infty}^{t} \mathrm{e}^{-L_{\omega}(t-s)} P_{2}^{\omega}\left(G_{\rho}(w(s))+K_{\omega}\right) \mathrm{d} s .
\end{aligned}
$$

We now prove Theorem 3.7.

Proof. We fix $\alpha, \beta, \eta$ to be as in the hypotheses of Proposition 3.5, and for $\rho$ small enough for $\left(C_{2}\right)$ to be realized, we apply Theorem 3.1. Then we know that there exists a global $C^{1}$ invariant manifold $\mathcal{M}_{\rho}$ for the flow (18), which satisfies $\left(G_{1}\right)$ and $\left(G_{2}\right)$. It is now sufficient to prove that $\mathcal{M}_{\rho}^{\text {loc }}$ is nonempty. With the Eq. (21), for $w_{0}=\zeta+h(\zeta) \in \mathcal{M}_{\rho}$, we have

$$
\left\|w\left(., w_{0}\right)\right\|_{C_{\eta}\left(\mathbb{R}^{-}, H_{0}^{1}(\omega)\right)} \leq 2 M|\zeta|_{L^{2}(\omega)}+2 K\left(\alpha, \beta, M, M^{*}\right)\left\|K_{\omega}\right\|_{L^{2}(\omega)}
$$

So for every $\zeta \in P_{1}^{\omega} L^{2}(\omega)$ such that

$$
\|\zeta\|_{L^{2}(\omega)}<\frac{\rho-2 K\left(\alpha, \beta, M, M^{*}\right)\left\|K_{\omega}\right\|_{L^{2}(\omega)}}{2 M},
$$

we infer that $\left\|w\left(0, w_{0}\right)\right\|_{H_{0}^{1}(\omega)}<\rho$, and then $\mathcal{M}_{\rho}^{l o c}$ is not empty.

REMARK 3.10. Let us make three remarks about this theorem.

1. In the proof, when we show that $\mathcal{M}_{\rho}^{\text {loc }}$ is nonempty, we prove actually that

$$
P_{1}^{\omega}\left(B_{L^{2}(\omega)}(0, r)\right) \subset P_{1}^{\omega} \mathcal{M}_{\rho}^{l o c}
$$

with $r=\frac{\rho-2 K\left(\alpha, \beta, M, M^{*}\right)\left\|K_{\omega}\right\|_{L^{2}(\omega)}}{2 M}$.

2. We can replace the condition $\left(C_{2}\right)$ by: $\operatorname{Lip}\left(G_{\rho}\right) K\left(\alpha, \beta, M, M^{*}\right)<1$. In this case, $\left(C_{3}\right)$ becomes $\left\|K_{\omega}\right\|_{L^{2}(\omega)}<\frac{\rho(1-\theta)}{K\left(\alpha, \beta, M, M^{*}\right)}$ where $\theta$ is such that one has $\operatorname{Lip}\left(G_{\rho}\right) K\left(\alpha, \beta, M, M^{*}\right)<\theta<1$. We have then the same result. In this theorem, we choose $\theta=\frac{1}{2}$ that is less general, but does not change the final result and simplifies the notation.

3. For every two-part spectral decomposition of $L_{\omega}$ such that $\lambda_{l}\left(L_{\omega}\right)>0$ (see (15)), there exists a family of global invariant manifolds $\mathcal{M}_{\rho}$ of the flow (18), which depends of $\rho$. And for each decomposition and each $\rho$ smaller enough, there exists a unique local manifold $\mathcal{M}_{\rho}^{\text {loc }}$ if only the condition $\left(C_{3}\right)$ is realized.

The next part is devoted to the extension of the manifold. 


\subsection{Extension of the manifolds}

The manifold $\mathcal{M}_{\rho}$ is a subset of $H_{0}^{1}(\omega)$. This space becomes a closed subspace of $H_{0}^{1}(D)$ by trivial extension by zero outside $\omega$. We wish that solutions of (21) were fixed points of a contraction in $C_{\eta}\left(\mathbb{R}^{-}, H_{0}^{1}(D)\right)$. In this aim, we introduce the mapping

$$
\mathcal{J}_{\omega}^{p}: Q_{1}^{\omega} L^{2}(D) \times C_{\eta}\left(\mathbb{R}^{-}, H_{0}^{1}(D)\right) \mapsto C_{\eta}\left(\mathbb{R}^{-}, H_{0}^{1}(D)\right)
$$

defined by

$$
\begin{aligned}
\mathcal{J}_{\omega}^{p}(\zeta, u)(t)= & T_{\omega}(t) \zeta+\int_{0}^{t} T_{\omega}(t-s) Q_{1}^{\omega}\left(G_{\rho}^{p}(u(s))+p\left(K_{\omega}\right)\right) \mathrm{d} s \\
& +\int_{-\infty}^{t} T_{\omega}(t-s) Q_{2}^{\omega}\left(G_{\rho}^{p}(u(s))+p\left(K_{\omega}\right)\right) \mathrm{d} s
\end{aligned}
$$

where $G_{\rho}^{p}$ is a mapping from $H_{0}^{1}(D)$ into $L^{2}(D)$ defined by:

$$
G_{\rho}^{p}(u)=\psi\left(\|u\|_{H_{0}^{1}(D)}\right)\left[f(u+p(\bar{v}))-f(p(\bar{v}))-f^{\prime}(p(\bar{v})) x\right] .
$$

This mapping is Lipschitz continuous and verifies

$$
\forall u \in L^{2}(\omega),\left.\quad G_{\rho}^{p}(p(u))\right|_{\omega}=G_{\rho}(u) .
$$

So, we have

$$
\operatorname{Lip}\left(G_{\rho}\right) \leq \operatorname{Lip}\left(G_{\rho}^{p}\right) .
$$

It follows that $\mathcal{J}_{\omega}^{p}$ is a contraction in $C_{\eta}\left(\mathbb{R}^{-}, H_{0}^{1}(D)\right)$ with a contraction coefficient smaller than $\operatorname{Lip}\left(G_{\rho}^{p}\right) K\left(\alpha, \beta, M, M^{*}\right)$. We also have the following lemma.

LEMMA 3.11. For all $\rho>0$ and all $n \in \mathbb{N}$, the mappings $G_{\rho}$ and $G_{\rho}^{n}$ belong to $C^{1}\left(H_{0}^{1}(D), L^{2}(D)\right)$. Moreover, for all $\varepsilon>0$, there exist $\rho>0$ and $N_{\rho}$, which depend only on $\rho$ such that

$$
\left\{\begin{aligned}
\operatorname{Lip}\left(G_{\rho}\right) & \leq \varepsilon, \\
\forall n>N_{\rho}, & \operatorname{Lip}\left(G_{\rho}^{n}\right) \leq \varepsilon
\end{aligned}\right.
$$

Proof (of Lemma 3.11). To prove the first part of the lemma, it is sufficient to show that $f \in C^{1}\left(H_{0}^{1}(D), L^{2}(D)\right) . f$ is Gâteau-differentiable from $L^{2}(D)$ into $L^{2}(D)$. Indeed, $\left|\frac{f(u+t \varphi)-f(u)}{t}-f^{\prime}(u) \varphi\right|$ converge a.e. to 0 as $t$ goes to 0 and are bounded by $2 c|\varphi|$. So,

$$
\left\|f(u+t \varphi)-f(u)-f^{\prime}(u) t \varphi\right\|_{L^{2}(D)}=o(t) .
$$

We prove by contradiction that $f^{\prime}$ is a continuous mapping from $H_{0}^{1}(D)$ into $\left.\mathcal{L}\left(H_{0}^{1}(D), L^{2}(D)\right)\right)$. Suppose there exists two sequences $\left(u_{n}\right)_{n \in \mathbb{N}}$ and $\left(h_{n}\right)_{n \in \mathbb{N}}$ such that $u_{n} \stackrel{H_{0}^{1}(D)}{\longrightarrow} u$, for all $n \in \mathbb{N},\left\|h_{n}\right\|_{H_{0}^{1}(D)} \leq 1$ and

$$
\left\|\left(f^{\prime}\left(u_{n}\right)-f^{\prime}(u)\right) h_{n}\right\|_{L^{2}(D)}>\delta>0 .
$$


By the compact embedding of $H_{0}^{1}(D)$ into $L^{2}(D),\left(f^{\prime}\left(u_{n}\right)-f^{\prime}(u)\right) h_{n}$ converges, up to a subsequence, a.e. to zero and is uniformly bounded by a $L^{2}(D)$ function. (25) is then a contradiction with the dominated convergence theorem. Using the same argument, we show that $f^{\prime}$ is uniformly continuous in the bounded subset of $H_{0}^{1}(D)$, and so, for all $\varepsilon>0$ there exists $\rho>0$ and $N_{\rho}$, which depend only on $\rho$ such that for all $v \in B_{H_{0}^{1}(D)}(0, \rho)$

$$
\begin{aligned}
\sup _{B(0, \rho)}\left\|g^{\prime}(v)\right\|_{\mathcal{L}\left(H_{0}^{1}(D), L^{2}(D)\right)} & <\varepsilon, \\
\text { and } \forall n>N_{\rho}, & \sup _{B(0, \rho)}\left\|g_{n}^{\prime}(v)\right\|_{\mathcal{L}\left(H_{0}^{1}(D), L^{2}(D)\right)}<\varepsilon .
\end{aligned}
$$

Here, as in the rest of the proof, $B(0, r)$ is the ball in $H_{0}^{1}(D)$. Now if we compute $G_{\rho}^{\prime}$, we obtain for $v, h \in H_{0}^{1}(D)$

$$
G_{\rho}^{\prime}(v) h=\frac{((x, h))}{\|v\|_{H_{0}^{1}(D)}} \frac{1}{\rho} \psi^{\prime}\left(\frac{\|v\|_{H_{0}^{1}(D)}}{\rho}\right) g(v)+\psi_{\rho}\left(\|v\|_{H_{0}^{1}(D)}\right) g^{\prime}(v) h .
$$

We infer that

$$
\begin{aligned}
\left\|G_{\rho}^{\prime}(v)\right\|_{\mathcal{L}\left(H_{0}^{1}(D), L^{2}(D)\right) \leq} & \frac{\sup \left|\psi^{\prime}\right|}{\rho} \sup _{B(0,2 \rho)}\|g(v)\|_{L^{2}(D)} \\
& +\sup _{B(0,2 \rho)}\left\|g^{\prime}(x)\right\|_{\mathcal{L}\left(H_{0}^{1}(D), L^{2}(D)\right)}
\end{aligned}
$$

If we choose $\rho$ such that $\left\|g^{\prime}(v)\right\|_{\mathcal{L}\left(H_{0}^{1}(D), L^{2}(D)\right)}<\varepsilon\left(1+2 \sup \left|\psi^{\prime}\right|\right)$, as $g(0)=0$, we have $\sup _{B(0,2 \rho)}\|g(v)\|_{L^{2}(D)} \leq \varepsilon\left(1+2 \sup \left|\psi^{\prime}\right|\right)$. We thus obtain

$$
\sup _{H_{0}^{1}(D)}\left\|G_{\rho}^{\prime}(v)\right\|_{\mathcal{L}\left(H_{0}^{1}(D), L^{2}(D)\right)}<\varepsilon .
$$

Let now $N_{\rho}$ be such that for all $n>N_{\rho},\left\|g_{n}^{\prime}(v)\right\|_{\mathcal{L}\left(H_{0}^{1}(D), L^{2}(D)\right)}<\varepsilon\left(1+2 \sup \left|\psi^{\prime}\right|\right)$ then for $n>N_{\rho}$ we have also

$$
\sup _{H_{0}^{1}(D)}\left\|G_{\rho}^{n \prime}(v)\right\|_{\mathcal{L}\left(H_{0}^{1}(D), L^{2}(D)\right)}<\varepsilon
$$

and the result follows.

Now, let $\varphi \in C_{\eta}\left(\mathbb{R}^{-}, H_{0}^{1}(\omega)\right)$. We have for all $t \leq s \leq 0$ if $i=1$ and all $s \leq t \leq 0$ if $i=2$

$$
T_{\omega}(t-s) Q_{i} G_{\rho}^{p}(p(\varphi(s)))=p\left[\mathrm{e}^{-L_{\omega}(t-s)} P_{i} G^{\rho}(\varphi(s))\right] .
$$

We infer that for every $\zeta \in Q_{1}^{\omega} L^{2}(D)$,

$$
\mathcal{J}_{\omega}^{p}(\zeta, p(\varphi))=p\left(\mathcal{J}_{\omega}\left(\left.\zeta\right|_{\omega}, \varphi\right)\right)
$$


Thanks to (24), if $\mathcal{J}_{\omega}^{p}$ is strictly contractive, then $\mathcal{J}_{\omega}$ is also strictly contractive. Hence, by Lemma 3.11 and since for every $\zeta \in Q_{1}^{\omega} L^{2}(D)$ we have $\mathcal{J}_{\omega}^{p}(\zeta, p(\varphi))=$ $\mathcal{J}_{\omega}^{p}\left(p\left(\left.\zeta\right|_{\omega}\right), p(\varphi)\right)$, we conclude that the unique fixed point of $\mathcal{J}_{\omega}^{p}(\zeta,$.$) is p(\varphi)$, where $\varphi$ is the fixed point of $\mathcal{J}_{\omega}\left(\left.\zeta\right|_{\omega}\right.$, . ). Consequently, $\mathcal{M}_{\rho}$ considered as a subset of $H_{0}^{1}(D)$ is equal to $\left\{\varsigma+H_{\omega}(\varsigma), \varsigma \in Q_{1}^{\omega} L^{2}(D)\right\}$, where $H_{\omega}(\varsigma)=p\left(h_{\omega}\left(\left.\varsigma\right|_{\omega}\right)\right)$ and $\varsigma+H_{\omega}(\varsigma)$ is the fixed point of $\mathcal{J}^{p}(\varsigma,$.$) at time t=0$. The local manifold $\mathcal{M}_{\rho}^{\text {loc }}$ in $H_{0}^{1}(D)$ is then defined by

$$
\mathcal{M}_{\rho}^{l o c}=\mathcal{M}_{\rho} \cap B_{H_{0}^{1}(D)}(0, \rho) .
$$

In the following section, we will omit the symbol $p$ to simplify the notations, but we always work in the functional space defined on $D$. The mapping $G_{\rho}$ will be always replaced by the mapping $G_{\rho}^{p}$, it does not involve any problem because the condition $\left(C_{2}\right)$ occurs for $G_{\rho}^{p}$ as soon as it occurs for $G_{\rho}$.

\section{Stability of the manifolds}

In what follows, $\bar{u} \in S P(\Omega)$. We denote by $L$ the operator $L_{\Omega}=-\Delta_{\Omega}-f^{\prime}(\bar{u})$ with the domain $D(L)=D\left(-\Delta_{\Omega}\right)$. The main result of this paper is the following theorem.

THEOREM 4.1. Let $\left(\Omega_{n}\right)_{n \in \mathbb{N}}$ be a sequence of open sets in $\mathcal{O}(D)$ which converges in the sense of Mosco to $\Omega$ and $\bar{u} \in S P(\Omega)$. Then for all $l \in \mathbb{N}$ such that

$$
\lambda_{l-1}(L)<\lambda_{l}(L) \text { and } 0<\lambda_{l}(L),
$$

there exists a $(l-1)$-dimensional local invariant manifold $\mathcal{M}$ of $P_{\Omega}$ that contains $\bar{u}$. For $n$ large enough, $P_{\Omega_{n}}$ has also a $(l-1)$-dimensional local invariant manifold $\mathcal{M}_{n}$. Moreover, there exists $\rho_{0}>0$ such that

$$
\lim _{n \rightarrow \infty} d_{B\left(\bar{u}, \rho_{0}\right)}\left(\mathcal{M}_{n}, \mathcal{M}\right)=0 .
$$

REMARK 4.2. If $\bar{u}$ is a nonhyperbolic point and if we choose $l$ in such a way that $\lambda_{l-1}(L)=0$, the manifold $\mathcal{M}$ is a central unstable manifold for the flow of $P_{\Omega}$.

\subsection{Proof of Theorem 4.1: outline}

We will prove this result in two steps. In the first one, we show the existence of local manifolds of $P_{\Omega}$ and $P_{\Omega_{n}}$. In the second one, we prove the convergence result. Step 1: existence. Let $a, b$ be two nonnegative real numbers such that

$$
\lambda_{l-1}(L)<a<b<\lambda_{l}(L)
$$

and let $\alpha, \beta, \eta$ be real numbers verifying the hypotheses of Proposition 3.5. Now $M$ and $M^{*}$ are fixed. We apply Theorem 3.7 at $\omega=\Omega$ and $\bar{v}=\bar{u}$. There exists $\rho_{0}$ such 
that the condition $\left(C_{2}\right)$ is satisfied (see Lemma 3.11). So there exists $\mathcal{M}_{\rho_{0}}(l-1)$ dimensional global manifold of the flow $(18)$, which satisfies $\left(G_{1}\right)$ and $\left(G_{2}\right)$. We will note in what follows $H$ instead of $H_{\Omega}$. Since $\bar{u}$ is a fixed point $\left(K_{\Omega}=0\right)$, the condition $\left(C_{3}\right)$ is obviously satisfied and $0 \in \mathcal{M}_{\rho_{0}}$. We then conclude that $\mathcal{M}$, defined by

$$
\mathcal{M}=\bar{u}+\mathcal{M}_{\rho_{0}}^{l o c}
$$

is a $(l-1)$-dimensional local invariant manifold of $P_{\Omega}$ such that $\bar{u} \in \mathcal{M}$. Let us now look at the flow on $\Omega_{n}$. There does not exist necessarily a sequence $\bar{u}_{n} \in \operatorname{SP}\left(\Omega_{n}\right)$ such that $\bar{u}_{n} \stackrel{H_{0}^{1}(D)}{\longrightarrow} \bar{u}$, but we have the following result.

LEMMA 4.3. Let $\left(\Omega_{n}\right)_{n \in \mathbb{N}}$ be a sequence of open sets in $\mathcal{O}(D)$, converging in the sense of Mosco to an open set $\Omega$. Let $\bar{u} \in D\left(-\Delta_{\Omega}\right)$ such that $-\Delta_{\Omega} \bar{u}=f(\bar{u})$. Then there exists $\bar{u}_{n} \in D\left(-\Delta_{\Omega_{n}}\right)$ such that:

- $\bar{u}_{n} \stackrel{H_{0}^{1}(D)}{\longrightarrow} \bar{u}$

- $\left\|-\Delta_{\Omega_{n}} \bar{u}_{n}-f\left(\bar{u}_{n}\right)\right\|_{L^{2}\left(\Omega_{n}\right)} \rightarrow 0$.

Proof (of Lemma 4.3). The convergence in the sense of Mosco implies existence of a sequence of functions $v_{n} \in H_{0}^{1}\left(\Omega_{n}\right)$ such that $v_{n} \stackrel{H_{0}^{1}(D)}{\longrightarrow} \bar{u}$. For all $n$, let $\bar{u}_{n} \in$ $H_{0}^{1}\left(\Omega_{n}\right)$ be the unique solution in the sense of the distributions in $\Omega_{n}$ of

$$
-\Delta \bar{u}_{n}=f\left(v_{n}\right) \text {. }
$$

For all the sequence $\left(v_{n}\right)_{n \in \mathbb{N}}$, we have $f\left(v_{n}\right) \stackrel{L^{2}(D)}{\longrightarrow} f(\bar{u})$, and by passing to the limit in the equation above we infer that $\left(\bar{u}_{n}\right)_{n \in \mathbb{N}}$ converges in $H_{0}^{1}(D)$ to $\bar{u}$. So, we also have $f\left(\bar{u}_{n}\right) \stackrel{L^{2}(D)}{\longrightarrow} f(\bar{u})$. But

$$
\begin{aligned}
\left\|-\Delta_{\Omega_{n}} \bar{u}_{n}-f\left(\bar{u}_{n}\right)\right\|_{L^{2}\left(\Omega_{n}\right)} & =\left\|f\left(v_{n}\right)-f\left(\bar{u}_{n}\right)\right\|_{L^{2}\left(\Omega_{n}\right)} \\
& =\left\|f\left(v_{n}\right)-f\left(\bar{u}_{n}\right)\right\|_{L^{2}(D)}
\end{aligned}
$$

because $v_{n}=u_{n}$ a.e. outside $\Omega_{n}$. We infer the result.

Proof of Theorem 4.1, continued. Recall that $l, a, b, c, \eta, \alpha, \beta$ are fixed as $M, M^{*}$ and $K\left(\alpha, \beta, M, M^{*}\right)$. We replace $\omega$ by $\Omega_{n}$ and $\bar{v}$ by $\bar{u}_{n}$ in Sect. 3. For $n$ large enough, the condition $\left(C_{1}\right)$ is verified because the eigenvalues of $L_{\Omega_{n}}$ converge to the eigenvalues of $L$ : this result is stated below as Theorem 4.5). The condition $\left(C_{2}\right)$ is satisfied for $\rho_{0}$ by Lemma 3.11, and the condition $\left(C_{3}\right)$ is verified by Lemma 4.3 . Hence, for $n$ large enough, there exists a global invariant manifold $\mathcal{M}_{\rho_{0}}^{n}$ for the flow (18), satisfying $\left(G_{1}\right)$ and $\left(G_{2}\right)$, and also a local invariant manifold $\mathcal{M}_{\rho_{0}}^{\text {,loc }}$ for the flow (10) defined by

$$
\mathcal{M}_{\rho_{0}}^{n, l o c}=\mathcal{M}_{\rho_{0}}^{n} \cap B_{H_{0}^{1}(D)}\left(0, \rho_{0}\right) .
$$

The set $\mathcal{M}_{n}$ defined by

$$
\mathcal{M}_{n}=\bar{u}_{n}+\mathcal{M}_{\rho_{0}}^{n, l o c}
$$

is then a local invariant manifold of $P_{\Omega_{n}}$. 
Before showing convergence of the manifolds, we introduce some new notations. We note $L_{n}$ instead of $L_{\Omega_{n}}=-\Delta_{\Omega_{n}}-f^{\prime}\left(\bar{u}_{n}\right), T_{n}(t)$ for the degenerate semigroup of $L_{n}, Q_{i}^{n}$ instead of $Q_{i}^{\Omega_{n}}(i=1,2), G_{\rho}^{n}$ for the nonlinearity in the Eq. (18) and $K_{n}$ instead of $K_{\Omega_{n}}=-\Delta_{\Omega_{n}} \bar{u}_{n}-f\left(\bar{u}_{n}\right)$.

Step 2: convergence. Assume that the following lemma is proved: we postpone its proof to Sect. 4.3 .

LEMMA 4.4. Let $\left(\zeta_{n}\right)_{n \in \mathbb{N}}$ be sequence such that $\zeta_{n} \in Q_{1}^{n} H_{0}^{1}(D)$, converging in $H_{0}^{1}(D)$ to $\zeta$. Then $\zeta \in Q_{1} H_{0}^{1}(D)$ and

$$
H_{n}\left(\zeta_{n}\right) \stackrel{H_{0}^{1}(D)}{\longrightarrow} H(\zeta)
$$

In order to end the proof of Theorem 4.1, it is sufficient to show that for all $\rho<\rho_{0}$, we have $\varrho_{\rho}\left(\mathcal{M}_{n}, \mathcal{M}\right) \rightarrow 0$. We begin with showing that the first term in the maximization converges to zero. Let $\left(x_{n}\right)_{n \in \mathbb{N}}$ be a maximizing sequence, there exists $\zeta_{n} \in H_{0}^{1}(D)$ such that $\left\|\zeta_{n}\right\|_{H_{0}^{1}(D)} \leq \rho$ and such that $x_{n}=\bar{u}_{n}+\zeta_{n}+H_{n}\left(\zeta_{n}\right)$. Up to a subsequence, and thanks to the fact that $Q_{1}^{n}$ converge uniformly to $Q_{1}$ (Corrolary 4.7), there exists $\zeta \in Q_{1} H_{0}^{1}(D) \cap \bar{B}(0, \rho)$ such that $\zeta_{n} \stackrel{H_{0}^{1}(D)}{\longrightarrow} \zeta$. Then $x_{n} \stackrel{H_{0}^{1}(D)}{\longrightarrow} x=$ $\bar{u}+\zeta+H(\zeta)$ (Lemma 4.4), and since $x_{n} \in \bar{B}(\bar{u}, \rho)$, we have $x \in \mathcal{M}$. We conclude that $d\left(x_{n}, \mathcal{M}\right) \longrightarrow 0$. To prove that the second term also converges to zero, since $\mathcal{M} \cap \bar{B}(\bar{u}, \rho)$ is compact, it is sufficient to prove that for all $x \in \mathcal{M} \cap \bar{B}(\bar{u}, \rho)$ there exists $x_{n} \in \mathcal{M}_{n} \cap \bar{B}(\bar{u}, \rho)$ such that $x_{n} \stackrel{H_{0}^{1}(D)}{\longrightarrow} 0$. Let us write $x=\bar{u}+\zeta+H(\zeta)$ with $\zeta \in Q_{1} H_{0}^{1}(D)$, then it is easy to see that the sequence $\left(x_{n}\right)$ defined by $x_{n}=$ $\bar{u}_{n}+Q_{1}^{n}(\zeta)+H\left(Q_{1}^{n}(\zeta)\right)$ respects the two properties: $x_{n} \stackrel{H_{0}^{1}(D)}{\longrightarrow} x$ and for $n$ large enough, $x_{n} \in \mathcal{M}_{n}$.

The end of this section is devoted to the proof of results that are used in the proof of Theorem 4.1 .

\subsection{Fundamental stability results}

In this part, we will prove on one hand the theorem about the convergence of eigenvalues and eigenspaces that are used in the proof of the Theorem 4.1 and, on the other hand, we will prove various results about the convergence of the semigroup $\left(T_{n}(t)\right)$, which will be used in the proof of Lemma 4.4. We keep the notations and hypotheses of the previous part.

In the following Theorem 4.5, we state essential facts about spectral convergence on which we base our method. We refer, for example, to [15] for the proof (see Corollaries 4.3 and 4.7). Note also that we can find a proof of a similar theorem in [3,9]. Analogous results with different hypotheses on convergence are proven in [1], and in [2] for Neumann boundary conditions. 
THEOREM 4.5 .

$$
\begin{gathered}
\forall \lambda<\lambda_{1}-c \quad \lim _{n \rightarrow \infty}\left\|R\left(\lambda, L_{n}\right)-R(\lambda, L)\right\|_{\mathcal{L}\left(L^{2}(D)\right)}=0 . \\
\forall k \in \mathbb{N}, \quad \lambda_{k}\left(L_{n}\right) \longrightarrow \lambda_{k}(L) \text { as } n \rightarrow \infty .
\end{gathered}
$$

The next theorem gives stability of the semigroup $T(t)$ in finite time.

\section{THEOREM 4.6.}

$$
\forall \delta>0, \forall T>\delta \quad \sup _{t \in[\delta, T]}\left\|T_{n}(t)-T(t)\right\|_{\mathcal{L}\left(L^{2}(D), H_{0}^{1}(D)\right)} \stackrel{n \rightarrow+\infty}{\longrightarrow} 0 .
$$

In what follows, we will note

$$
\|\cdot\|_{2}=\|\cdot\|_{\mathcal{L}\left(L^{2}(D)\right)} \quad \text { and } \quad\|\cdot\|_{2,1}=\|\cdot\|_{\mathcal{L}\left(L^{2}(D), H_{0}^{1}(D)\right)} \cdot
$$

Proof. Convergence in the space $\mathcal{L}\left(L^{2}(D)\right)$ is given by [16, Theorem 6.1].

We reach the final result by the equicontinuity argument: let $\left(f_{n}\right)_{n \in \mathbb{N}}$ be a uniformly bounded sequence in $L^{2}(D)$. The sequence $\left(\left(T_{n}(t)-T(t) f_{n}\right)_{n \in \mathbb{N}}\right.$ converges in $C\left([\delta, T], L^{2}(D)\right)$ to zero. Thanks to $(12)$, this sequence is also uniformly bounded and equicontinuous in $C\left([\delta, T], H_{0}^{1}(D)\right)$, so it converges to zero in $C\left([\delta, T], H_{0}^{1}(D)\right)$. The result follows.

COROLLARY 4.7.

$$
\begin{aligned}
\forall \delta>0, \forall T>\delta & \sup _{t \in[\delta, T]}\left\|T_{n}(t) Q_{2}^{n}-T(t) Q_{2}\right\|_{\mathcal{L}\left(L^{2}(D), H_{0}^{1}(D)\right)} \stackrel{n \rightarrow+\infty}{\longrightarrow} 0, \\
& \sup _{t \in[-T, T]}\left\|T_{n}(t) Q_{1}^{n}-T(t) Q_{1}\right\|_{\mathcal{L}\left(L^{2}(D), H_{0}^{1}(D)\right)} \stackrel{n \rightarrow+\infty}{\longrightarrow} 0 .
\end{aligned}
$$

Proof. The first statement comes from the second one by the triangle inequality. Let us thus prove the second statement. Let $B$ be a ball in the complex plane containing uniquely the eigenvalues $\lambda_{i}(L)$ for $i$ from 1 to $l-1$. Then

$$
T(t) Q_{1}=\frac{1}{2 i \pi} \int_{\partial B} \mathrm{e}^{\lambda t} R(\lambda,-L) \mathrm{d} \lambda .
$$

By Theorem 4.5, this last equality is also true with $Q_{1}^{n}$ instead of $Q_{1}$ and $L_{n}$ instead of $L$ for $n$ large enough. The uniform convergence of resolvent operators implies that

$$
\sup _{t \in[-T, T]}\left\|T_{n}(t) Q_{1}^{n}-T(t) Q_{1}\right\|_{2} \stackrel{n \rightarrow+\infty}{\longrightarrow} 0 .
$$

The final result comes with the argument of equicontinuity as the one used in the proof of Theorem 4.6. 


\subsection{Proof of Lemma 4.4}

Proof. First of all, in Lemma 4.4, the fact that $\zeta \in Q_{1} H_{0}^{1}(D)$ is an immediate consequence of Corollary 4.7. Indeed, $Q_{1}^{n}$ converge uniformly to $Q_{1}$ so $Q_{1}^{n} \zeta_{n} \stackrel{L^{2}(D)}{\longrightarrow} Q_{1} \zeta$ and $\zeta=Q_{1} \zeta$. We show now a key result of the proof of (29). Recall that with the notation of Chow and $\mathrm{Lu}$, the operator $\mathcal{F}$ defined by

$$
\mathcal{F} f=\int_{0}^{t} T(t-s) Q_{1} f(s) \mathrm{d} s+\int_{-\infty}^{t} T(t-s) Q_{2} f(s) \mathrm{d} s
$$

is a bounded linear operator from $C_{\eta}\left(\mathbb{R}^{-}, L^{2}(D)\right)$ into $C_{\eta}\left(\mathbb{R}^{-}, H_{0}^{1}(D)\right)$ such that

$$
\|\mathcal{F}\| \leq K\left(\alpha, \beta, M, M^{*}\right) .
$$

We define $\mathcal{F}_{n}$ in the same way for the semigroup $T_{n}(t)$. Then we have the following result.

\section{LEMMA 4.8.}

$$
\lim _{n \rightarrow \infty}\left\|\mathcal{F}_{n}-\mathcal{F}\right\|_{\mathcal{L}\left(C_{\eta}\left(\mathbb{R}^{-}, L^{2}(D)\right), C_{\eta}\left(\mathbb{R}^{-}, H_{0}^{1}(D)\right)\right)}=0 .
$$

Proof. Let $\varphi \in C_{\eta}\left(\mathbb{R}^{-}, L^{2}(D)\right)$. Suppose that $t \leq 0$. For all $T, \delta$ such that $T>\delta>0$, with the estimate (8), we have

$$
\begin{aligned}
\mathrm{e}^{-\eta t} \int_{t-\delta}^{t}\left\|T_{n}(t-s) Q_{2}^{n}(\varphi(s))-T(t-s) Q_{2}(\varphi(s))\right\|_{H_{0}^{1}(D)} \mathrm{d} s \\
\leq 2 \int_{t-\varepsilon}^{t}\left[\frac{M}{\sqrt{t-s}}+M^{*}\right] \mathrm{e}^{-\beta(t-s)} \mathrm{e}^{-\eta s}\|\varphi(s)\|_{L^{2}(D)} \mathrm{d} s \\
\leq 2\left[M^{*} \delta+2 M \sqrt{\delta}\right]\|\varphi\|_{C_{\eta}\left(\mathbb{R}^{-}, L^{2}(D)\right)} \text { and }, \\
\mathrm{e}^{-\eta t} \int_{-\infty}^{t-T}\left\|T_{n}(t-s) Q_{2}^{n}(\varphi(s))-T(t-s) Q_{2}(\varphi(s))\right\|_{H_{0}^{1}(D)} \mathrm{d} s \\
\leq 2 \int_{-\infty}^{t-T}\left[\frac{M}{\sqrt{t-s}}+M^{*}\right] \mathrm{e}^{-\beta(t-s)} \mathrm{e}^{-\eta s}\|\varphi(s)\|_{L^{2}(D)} \mathrm{d} s \\
\leq 2\left[\frac{M}{\sqrt{T}}+M^{*}\right]\left(\frac{\mathrm{e}^{-\beta(T)}}{\beta}\right)\|\varphi\|_{C_{\eta}\left(\mathbb{R}^{-}, L^{2}(D)\right)} .
\end{aligned}
$$

By a change of variables $(y=t-s)$, we have also

$$
\begin{aligned}
& \mathrm{e}^{-\eta t} \int_{t-T}^{t-\delta}\left\|T_{n}(t-s) Q_{2}^{n}(\varphi(s))-T(t-s) Q_{2}(\varphi(s))\right\|_{H_{0}^{1}(D)} \mathrm{d} s \\
& \leq \int_{t-T}^{t-\delta} \mathrm{e}^{-\eta y}\left\|T_{n}(y) Q_{2}^{n}-T(y) Q_{2}\right\|_{2,1} \mathrm{e}^{t-y}\|\varphi(t-y)\|_{L^{2}(D)} \mathrm{d} y \\
& \leq \frac{\mathrm{e}^{-\eta T}}{-\eta} \sup _{y \in[\delta, T]}\left\|T_{n}(y) Q_{2}^{n}-T(y) Q_{2}\right\|_{2,1}\|\varphi\|_{C_{\eta}\left(\mathbb{R}^{-}, L^{2}(D)\right)} .
\end{aligned}
$$


For all $\varepsilon>0$, there exists $\delta$ and $T(T>\delta>0)$ such that

$$
M^{*} \delta+2 M \sqrt{\delta}<\frac{\varepsilon}{6}, \quad\left[\frac{M}{\sqrt{T}}+M^{*}\right]\left(\frac{\mathrm{e}^{-\beta T}}{\beta}\right)<\frac{\varepsilon}{6} .
$$

Using Corollary 4.7, we obtain for $n$ large enough

$$
\begin{aligned}
& \sup _{\mathbb{R}^{-}} \mathrm{e}^{-\eta t} \int_{-\infty}^{t}\left\|T_{n}(t-s) Q_{2}^{n}(\varphi(s))-T(t-s) Q_{2}(\varphi(s))\right\|_{H_{0}^{1}(D)} \mathrm{d} s \\
& \quad \leq \varepsilon\|\varphi\|_{C_{\eta}\left(\mathbb{R}^{-}, L^{2}(D)\right)} .
\end{aligned}
$$

Now for all $T>0$ such that $t<-T$, we have with the estimate (6)

$$
\begin{aligned}
& \mathrm{e}^{-\eta t} \int_{t+T}^{0}\left\|T_{n}(t-s) Q_{1}^{n}(\varphi(s))-T(t-s) Q_{1}(\varphi(s))\right\|_{H_{0}^{1}(D)} \mathrm{d} s \\
& \quad \leq 2 M \int_{t+T}^{0} \mathrm{e}^{\alpha(t-s)} \mathrm{e}^{-\eta s}\|\varphi(s)\|_{L^{2}(D)} \mathrm{d} s \\
& \quad \leq 2 M \frac{\mathrm{e}^{-\alpha T}-\mathrm{e}^{\alpha t}}{\alpha}\|\varphi\|_{C_{\eta}\left(\mathbb{R}^{-}, L^{2}(D)\right)}, \quad \text { and } \\
& \mathrm{e}^{-\eta t} \int_{t}^{t+T}\left\|T_{n}(t-s) Q_{1}^{n}(\varphi(s))-T(t-s) Q_{1}(\varphi(s))\right\|_{H_{0}^{1}(D)} \mathrm{d} s \\
& \quad \leq \int_{-T}^{0} \mathrm{e}^{-\eta y}\left\|T_{n}(y) Q_{1}^{n}-T(y) Q_{1}\right\|_{2,1} \mathrm{e}^{t-y}\|\varphi(t-y)\|_{L^{2}(D)} \mathrm{d} y \\
& \quad \leq \frac{1}{-\eta} \sup _{y \in[-T, 0]}\left\|T_{n}(y) Q_{1}^{n}-T(y) Q_{1}\right\|_{2,1}\|\varphi\|_{C_{\eta}\left(\mathbb{R}^{-}, L^{2}(D)\right)} .
\end{aligned}
$$

For $\varepsilon>0$, let $T>0$ be such that $M \frac{\mathrm{e}^{-\alpha T}}{\alpha}<\frac{\varepsilon}{6}$ then for all $t<-T$

$$
\begin{aligned}
\mathrm{e}^{-\eta t} & \int_{t}^{0}\left\|T_{n}(t-s) Q_{1}^{n}(\varphi(s))-T(t-s) Q_{1}(\varphi(s))\right\|_{H_{0}^{1}(D)} \mathrm{d} s \\
\leq & {\left[\frac{2 \varepsilon}{3}+\sup _{y \in[-T, 0]}\left\|T_{n}(y) Q_{1}^{n}-T(y) Q_{1}\right\|_{2,1}\right]\|\varphi\|_{C_{\eta}\left(\mathbb{R}^{-}, L^{2}(D)\right)} . }
\end{aligned}
$$

But we have also for $t \geq-T$

$$
\begin{aligned}
& \mathrm{e}^{-\eta t} \int_{t}^{0}\left\|T_{n}(t-s) Q_{1}^{n}(\varphi(s))-T(t-s) Q_{1}(\varphi(s))\right\|_{H_{0}^{1}(D)} \mathrm{d} s \\
& \quad \leq \sup _{y \in[t, 0]}\left\|T_{n}(y) Q_{1}^{n}-T(y) Q_{1}\right\|_{2,1}\|\varphi\|_{C_{\eta}\left(\mathbb{R}^{-}, L^{2}(D)\right)} \\
& \quad \leq \sup _{y \in[-T, 0]}\left\|T_{n}(y) Q_{1}^{n}-T(y) Q_{1}\right\|_{2,1}\|\varphi\|_{C_{\eta}\left(\mathbb{R}^{-}, L^{2}(D)\right)} .
\end{aligned}
$$

So, for $n$ large enough with Corollary 4.7, we obtain

$$
\begin{aligned}
& \sup _{\mathbb{R}^{-}} \mathrm{e}^{-\eta t} \int_{t}^{0}\left\|T_{n}(t-s) Q_{1}^{n}(\varphi(s))-T(t-s) Q_{1}(\varphi(s))\right\|_{H_{0}^{1}(D)} \mathrm{d} s \\
& \quad \leq \varepsilon\|\varphi\|_{C_{\eta}\left(\mathbb{R}^{-}, L^{2}(D)\right)} .
\end{aligned}
$$

We finish the proof by combining (30) and (31). 
We can now finish the proof of Lemma 4.4. We note $\mathcal{J}$ instead of $\mathcal{J}_{\Omega}$ and $\mathcal{J}_{n}$ instead of $\mathcal{J}_{\Omega_{n}}$. It is sufficient to show that the sequence of fixed points of $\mathcal{J}_{n}\left(\zeta_{n}\right.$, .) converges in $C_{\eta}\left(\mathbb{R}^{-}, H_{0}^{1}(D)\right)$ to the fixed point of $\mathcal{J}(\zeta,$.$) . As \left(\mathcal{J}_{n}\left(\zeta_{n},.\right), \mathcal{J}(\zeta,).\right)$ are uniformly contractive-see Sect. 3.3-it is sufficient to apply Lemma 4.9 below.

LEMMA 4.9. For all $\varphi \in C_{\eta}\left(\mathbb{R}^{-}, H_{0}^{1}(D)\right)$

$$
J_{n}\left(\zeta_{n}, \varphi\right) \stackrel{C_{\eta}\left(\mathbb{R}^{-}, H_{0}^{1}(D)\right)}{\longrightarrow} J(\zeta, \varphi) .
$$

Proof. With the triangle inequality, we have

$$
\begin{aligned}
& \left\|\mathcal{J}_{n}\left(\zeta_{n}, \varphi\right)-\mathcal{J}(\zeta, \varphi)\right\|_{C_{\eta}\left(\mathbb{R}^{-}, H_{0}^{1}(D)\right)} \\
& \leq\left\|T_{n}(t) \zeta_{n}-T(t) \zeta\right\|_{C_{\eta}\left(\mathbb{R}^{-}, H_{0}^{1}(D)\right)} \\
& \quad+\left\|\mathcal{F}_{n}\left(G_{\rho}^{n}(\varphi)-G_{\rho}(\varphi)+K_{n}\right)\right\|_{C_{\eta}\left(\mathbb{R}^{-}, H_{0}^{1}(D)\right)} \\
& \quad+\left\|\left(\mathcal{F}_{n}-\mathcal{F}\right) G_{\rho}(\varphi)\right\|_{C_{\eta}\left(\mathbb{R}^{-}, H_{0}^{1}(D)\right)} .
\end{aligned}
$$

The first term on the right-hand side converges to zero by Corollary 4.7 and the estimate (6). For the second term, we need to show that $G_{\rho}^{n}(\varphi)$ converges to $G_{\rho}(\varphi)$ in $C_{\eta}\left(\mathbb{R}^{-}, L^{2}(D)\right)$. We have

$$
\begin{aligned}
& \left\|G_{\rho}^{n}(\varphi)-G_{\rho}(\varphi)\right\|_{C_{\eta}\left(\mathbb{R}^{-}, L^{2}(D)\right)} \leq\left\|g_{n}(\varphi)-g(\varphi)\right\|_{C_{\eta}\left(\mathbb{R}^{-}, L^{2}(D)\right)} \\
& \quad \leq 2 \operatorname{Lip} f\left\|\bar{u}_{n}-\bar{u}\right\|_{L^{2}(D)}+\left\|f^{\prime}\left(\bar{u}_{n}\right)-f^{\prime}(\bar{u})\right\|_{\mathcal{L}\left(H_{0}^{1}(D), L^{2}(D)\right)}|\varphi|_{C_{\eta}\left(\mathbb{R}^{-}, H_{0}^{1}(D)\right)} .
\end{aligned}
$$

The convergence follows because $f \in C^{1}\left(H_{0}^{1}(D), L^{2}(D)\right)$. For the last term, we apply Lemma 4.8 because $\left\|G_{\rho}(\varphi)\right\|_{C_{\eta}\left(\mathbb{R}^{-}, L^{2}(D)\right)} \leq 2 c\|\varphi\|_{C_{\eta}\left(\mathbb{R}^{-}, H_{0}^{1}(D)\right)}$, and the proof is finished.

\section{Some remarks}

1. Suppose that the steady-state point $\bar{u}$ is hyperbolic. By adapting to our domain perturbation the proof of Dancer [13, Theorem 1] based on the index theory of Leray-Schauder [23], we obtain existence of a sequence $\left(\bar{u}_{n}\right)_{n \in \mathbb{N}}$, which converges in $H_{0}^{1}(D)$ to $\bar{u}$ and such that for $n$ large enough $\bar{u}_{n} \in S P\left(\Omega_{n}\right)$ and is hyperbolic. Another way to prove existence of this sequence is to note that if $\bar{u}$ is hyperbolic, then 0 is a locally exponentially stable steady-state point of the flow defined by the equation

$$
\frac{\mathrm{d} z}{\mathrm{~d} t}+L\left(P_{2}-P_{1}\right) z=P_{2} g(z)-P_{1} g(z),
$$

where, following the notation of the previous part, $L$ is the operator $-\Delta_{\Omega}-f^{\prime}(\bar{u})$ and $P_{1}$ the associated projector for the nonpositive eigenvalues of $L$. If $\left(\bar{u}_{n}\right)_{n \in \mathbb{N}}$ is the sequence given by Lemma 4.3 , then the solutions of the equation

$$
\frac{\mathrm{d} z}{\mathrm{~d} t}+L_{n}\left(P_{2}^{n}-P_{1}^{n}\right) z=\left(P_{2}^{n}-P_{1}^{n}\right)\left(g_{n}(z)+K_{n}\right)
$$


with an initial condition in a neighborhood of zero are bounded. So, we infer existence of a fixed point to (34), which actually belongs to $S P(\Omega)$. Moreover this sequence of fixed points converges to $\bar{u}$.

We are now interested in the invariant manifolds in the case of a hyperbolic point. Let $l$ be such that $\lambda_{l}(L)$ is the first nonnegative eigenvalue of $L$. Then there exists $\varepsilon>0$ such that

$$
\lambda_{l-1}(L)<-\varepsilon<0<\lambda_{l}(L)
$$

Let us choose the constants $a, b, c, \eta, \alpha, \beta$ so that the hypotheses of Proposition 3.5 are satisfied, and also so that

$$
a<-\eta<\varepsilon<b \text { and } \sup \left|f^{\prime}\right|+\varepsilon<c .
$$

This choice gives us fixed constants $M, M^{*}$ and $K\left(\alpha, \beta, M, M^{*}\right)$. Moreover, the hypothesis (35) implies that the operator $L+\varepsilon$ verifies the estimates of $\left(H_{5}\right)$ with the same constants. Indeed, this operator can be written as $-\Delta_{\Omega}+c(x)$ with $|c(x)| \leq c$ and

$$
\lambda_{l-1}(L+\varepsilon)<a<b<\lambda_{l}(L+\varepsilon)
$$

We infer the estimates of $\left(H_{5}\right)$ for $L$ with $\theta:=\varepsilon+\eta$ - which is positive-instead of $\eta$. Let $\rho_{0}$ be such that $\left(C_{2}\right)$ is satisfied, using these estimates for the semigroup in the integral Eq. (21), we obtain that for all $w_{0} \in \mathcal{M}_{\rho_{0}}$ and all $t \leq 0$

$$
\|w(t)\|_{H_{0}^{1}(D)} \leq 2 M\left\|w_{0}\right\|_{L^{2}(D)} \mathrm{e}^{\theta t} .
$$

Following the definition of Hale [17], the unstable manifold $\mathcal{W}_{\omega}^{u}(\bar{u})$ of $P_{\omega}$ at a point $\bar{u}$ is defined by

$$
\begin{array}{r}
\mathcal{W}_{\omega}^{u}(\bar{u})=\left\{x_{0} \in L^{2}(D) / u\left(t, x_{0}\right) \text { exists for } t \leq 0\right. \\
\text { and } \left.u\left(t, x_{0}\right) \rightarrow 0 \text { as } t \rightarrow-\infty\right\}
\end{array}
$$

And for a neighborhood $U$ of $\bar{u}$, we define

$$
\mathcal{W}_{\omega}^{u}(\bar{u}, U)=\left\{x_{0} \in \mathcal{W}_{\omega}^{u}(\bar{u}): u\left(t, x_{0}\right) \in U, t \leq 0\right\},
$$

the unstable local manifold of $P_{\omega}$ at the point $\bar{u}$ with respect to the neighborhood $U$-the fact that the manifold is defined with respect to $U$ implies uniqueness of this manifold. Denoting by $B$ the ball $B_{H_{0}^{1}(D)}\left(\bar{u}, \frac{\rho_{0}}{2 M}\right)$, we obtain for $P_{\Omega}$

$$
\left\{\bar{u}+\mathcal{M}_{\rho_{0}}^{l o c}\right\} \cap B=\mathcal{W}_{\Omega}^{u}(\bar{u}, B),
$$

where $\mathcal{M}_{\rho_{0}}^{\text {loc }}$ is the local manifold given by the Theorem 3.7. The same argument is valid for $\bar{u}_{n}$. For $n$ large enough, the estimate (36) is valid for all $w_{0} \in \mathcal{M}_{\rho_{0}}^{n}$, and so we have

$$
\left\{\bar{u}_{n}+\mathcal{M}_{\rho_{0}}^{n, l o c}\right\} \cap B_{n}=\mathcal{W}_{\Omega_{n}}^{u}\left(\bar{u}_{n}, B_{n}\right)
$$


where $B_{n}=B_{H_{0}^{1}(D)}\left(\bar{u}_{n}, \frac{\rho_{0}}{2 M}\right)$. Theorem 4.1 involves convergence of the local invariant manifolds:

$$
\lim _{n \rightarrow \infty} d_{B\left(\bar{u}, \frac{\rho_{0}}{2 M}\right)}\left(\mathcal{W}_{\Omega_{n}}^{u}\left(\bar{u}_{n}, B_{n}\right), \mathcal{W}_{\Omega}^{u}(\bar{u}, B)\right)=0
$$

If now $\bar{v}$ is a point of $D\left(\Delta_{\Omega}\right)$ different from $\bar{u}$ but close to it in $H_{0}^{1}(D)$ norm, and such that $\left\|-\Delta_{\Omega} \bar{v}-f(\bar{v})\right\|_{L^{2}(\Omega)}$ is close enough to zero to apply Theorem 3.7 to the pair $(\Omega, \bar{v})$. The conditions $\left(C_{1}, C_{2}, C_{3}\right)$ are realized with the previous constants $l, K\left(\alpha, \beta, M, M^{*}\right), \rho_{0}$. Then there exists $\rho<\rho_{0}$ such that

$$
\left\{\bar{v}+\mathcal{M}_{\rho_{0}}^{l o c}\right\} \cap B(\bar{v}, \rho)=\mathcal{W}_{\Omega}^{u}(\bar{u}, B) \cap B(\bar{v}, \rho) .
$$

This fact allows to say that even if the sequence $\left(\bar{u}_{n}\right)_{n \in \mathbb{N}}$ is not a sequence of hyperbolic points, locally in a neighborhood of $\bar{u}_{n}$, the manifold $\mathcal{M}_{n}$ given by Theorem 4.1 coincides with the unstable local manifold of the hyperbolic point.

2. In the general case (nonhyperbolic), there is no reason for the two manifolds $\mathcal{M}_{\rho}$ constructed at two very close points or two different $\rho_{0}$ to coincide.

3 . When $\bar{u}$ is not hyperbolic $\left(\lambda_{l-1}(L)=0\right)$, and there exists a sequence of hyperbolic points $\bar{u}_{n} \in H_{0}^{1}\left(\Omega_{n}\right)$ converging to $\bar{u}$, then the local invariant manifold $\bar{u}_{n}+\mathcal{M}_{\rho}^{n, l o c}$ does not necessarily coincide with the unstable local manifold $\mathcal{W}_{\Omega_{n}}^{u}\left(\bar{u}_{n}\right)$. It will be the case only if $\lambda_{l-1}\left(L_{n}\right)<0$.

Open Access. This article is distributed under the terms of the Creative Commons Attribution License which permits any use, distribution, and reproduction in any medium, provided the original author(s) and the source are credited.

\section{REFERENCES}

[1] ARENDT, W. Approximation of degenerate semigroups, Taiwanese Journal of Mathematics, 5 (2001), no. 2, 279-295.

[2] ARRIETA, J.M.; CARVAlHO, A.N. Spectral convergence and nonlinear dynamics of reaction-diffusion equations under perturbations of the domain, J. Differential Equations, 199 (2004), 143-178.

[3] Atтouch, H. Variational Convergence for Functions and Operators, Pitman (1984).

[4] BATES, P.W.; LU, K.; ZENG, C. Existence and Persistence of Invariant Manifolds for Semiflows in Banach Space Mem. Am. Math. Soc. bf 135, (1998), no. 645.

[5] BATES, P.W.; LU, K.; ZENG, C. Normally hyperbolic invariant manifolds for semiflow in a banach space. Differential equations and applications (Hangzhou, 1996), 22-29, International Press, Cambridge, MA, 1997.

[6] BAtes, P.W.; LU, K.; ZENG, C. Persistence of overflowing Manifolds for semiflow Communications on Pure and Applied Mathematics, Vol. LII, 0983-1046 (1999)

[7] Bucur, D. Characterization for the Kuratowski Limits of a Sequence of Sobolev Spaces, J. Diff. Eq., 151 (1999), 1-19.

[8] BuCuR D.; ZolÉSIO, J.P. N-Dimensional Shape Optimization under Capacitary Constraints, J. Differential Equations, 123 (1995), No. 2, 504-522.

[9] BuCUR, D.; ZOLÉSIO, J.P. Stabilité du spectre d'un opérateur elliptique par rapport au domaine, C. R. Acad. Sci. Paris Sér. I Math., 324 (1997), no. 2, 191-194.

[10] Buttazzo, G.; Dal Maso, G. Shape optimization for Dirichlet problems: relaxed formulation and optimality conditions, Appl. Math. Optim., 23 (1991), 17-49.

[11] CHOw, S.; LU, K. Invariant manifolds for flows in Banach spaces J. Differential Equations, 74 (1988), no. 2, 285-317. 
[12] DANCER, E. N. The effect of domain shape on the number of positive solutions of certain nonlinear equations, J. Differential Equations, 74 (1988), no. 1, 120-156.

[13] DANCER, E. N. The effect of domain shape on the number of positive solutions of certain nonlinear equations. II, J. Differential Equations, 87 (1990), no. 2, 316-339.

[14] DANERS, D. Domain perturbation for linear and nonlinear parabolic equations, J. Differential Equations, 129 (1996), no. 2, 358-402.

[15] DANERS, D. Dirichlet problems on varying domains, J. Differential Equations, 188 (2003), 591624.

[16] DANERS, D. Perturbation of semi-linear evolution equations under weak assumptions at initial time, J. Differential Equations, 210 (2005), 352-382.

[17] HalE, J.K. Asymptotic behavior of dissipative systems, AMS, Providence, Rhode island.

[18] HENRY, D. Geometric theory of semilinear parabolic equations, Springer, Berlin, 1981.

[19] Kostin, I.N. Relatively unstable invariant sets of nonlineat operators J. Mathematical Sciences 99, no. 1 (2000)

[20] Mosco, UMBERTo Convergence of convex sets and of solutions of variational inequalities, Advances in Math., 3 (1969), 510-585.

[21] Simondon, F. Domain perturbation for parabolic quasilinear problems Applied Analysis, 4 (2000), no. 1, 1-12.

[22] SVERAK, V. On optimal shape design, J. Math. Pures Appl., 72 (1993), 537-551.

[23] ZEIDLER, E. Nonlinear Functional Analysis and its Applications I, Springer, New York, Berlin, Heidelberg, Tokyo.

\author{
Nicolas Varchon \\ Interdisciplinary Centre for Mathematical \\ and Computational Modelling, \\ University of Warsaw, \\ Zwirki i Wigury 93, \\ 02-089 Warsaw, Poland \\ E-mail:varchon@icm.edu.pl
}

